\title{
Universal statistics of the critical depinning force of elastic systems in random media
}

\author{
C. J. Bolech and Alberto Rosso \\ Université de Genève, DPMC, 24 Quai Ernest Ansermet, CH-1211 Genève 4, Switzerland
}

(Dated: February $\left.2^{\text {nd }}, 2004\right)$

\begin{abstract}
We study the rescaled probability distribution of the critical depinning force of an elastic system in a random medium. We put in evidence the underlying connection between the critical properties of the depinning transition and the extreme value statistics of correlated variables. The distribution is Gaussian for all periodic systems, while in the case of random manifolds there exists a family of universal functions ranging from the Gaussian to the Gumbel distribution. Both of these scenarios are a priori experimentally accessible in finite, macroscopic, disordered elastic systems.

PACS numbers: 46.65.+g,64.60.Ht,68.45.Gd
\end{abstract}

The study of disordered elastic objects sheds light on the physics of numerous experimental systems, including domain walls in magnetic [1] or ferroelectric [2] materials, contact lines of liquid menisci on a rough substrate [3] and propagating cracks in solids [4]. All these are accurately modeled as elastic manifolds in the presence of randomness. A second class of disordered elastic systems is given by periodic structures such as charge density waves [5], vortex flux lines in type-II superconductors [6] and Wigner crystals 7]. It has been proven that the periodic nature of these structures carries to the same universal behavior as elastic interfaces in the presence of periodic disorder $[8,9]$.

A continuing challenge is to understand the response of these systems to an applied external drive per unit surface, $f$. Two regimes are present at zero temperature: whenever $f$ is smaller than certain critical threshold $f_{c}$ the interface is pinned, but beyond this point $\left(f>f_{c}\right)$ the system undergoes a depinning transition [8, 10, 11, 12, 13. and continues to move with a non-zero average velocity. Throughout all of the low temperature regime the dynamics is strongly influenced by the presence of this threshold. It is thus interesting to characterize $f_{c}$ in detail, and in particular, to study its sample-dependent fluctuations. We remark that the critical force is actually the biggest one that still pins the system. New insights shall then be expected from exploring the eventual connections with the theory of extreme value statistics.

In a way reminiscent to the central limit theorem for the sum of uncorrelated variables, there exists a fundamental result for the statistics of extremes [14, 15]. It states that only three limits for the maxima of independent identically distributed (iid) variables are possible: the Weibull family (for upper bounded variables), the Fréchet family (for power-law-tailed variables) or the Gumbel distribution (for exponential-tailed variables). However, due to the complicated dynamics of elastic systems, we expect that strong correlations should play a crucial role in determining the limiting distribution of its thresholds. Indeed, the depinning transition is an example of a critical phenomenon with well-determined universal exponents, which implies strong, long ranged, well- characterized correlations. The few results known related to extreme values of correlated variables concern short range or weak correlations [14, 16, 17, 18]. The opposite case has only been started to be discussed [19, 20, 21]. Here, we identify and study an example of extremes of strongly correlated variables with clear physical interest.

To pose the problem, let us consider a fixed disorder realization on a finite system. To every configuration of the elastic interface, $\{\alpha\}$, we associate a depinning force, $f_{d}^{\{\alpha\}}$, given by the smallest non-negative force so that at least one point of the interface has instant non-negative velocity [22, 23]. The critical force of the realization follows naturally:

$$
f_{c}^{r}=\max _{\{\alpha\}}\left\{f_{d}^{\{\alpha\}}\right\} .
$$

In this letter we will concentrate on the statistics of $f_{c}^{r}$. We shall identify the universal aspects of its distribution and stress the important differences between the periodic and random manifold cases. We shall find that whilst for periodic systems the distribution is always a Gaussian, for random manifolds it belongs to a family that interpolates between the Gaussian and the Gumbel 34].

The focus of our attention will be a $d$-dimensional interface propagating transversally on a $(d+1)$-dimensional space; for clarity we describe now the case of a line $(d=1)$. At any given time, the line defines a singlevalued function $h(x)$, where $x$ is the longitudinal coordinate. Its zero temperature overdamped dynamics is governed by the following equation of motion,

$$
\partial_{t} h(x, t)=-\frac{\partial E(\{h, x\})}{\partial h(x, t)}=c \nabla^{2} h+f+\eta(x, h(x, t)) .
$$

The functional $E(\{h, x\})$ represents the total energy, including the harmonic elastic energy and the potential energy terms due to the drive $f$ and the short-range correlated disorder force $\eta(x, h)$. This is a non-linear equation whose analytic solution is unknown. As alluded to in the beginning, properties of the disorder distinguish two different cases. If $\eta(x, h)$ is a periodic function, Eq. (2) describes a periodic system, whereas the random manifolds are characterized by a non-periodic disorder. 
Our approach to studying Eq. (2) will be numerical. In particular, for each disorder realization, we find the critical force $f_{c}^{r}$ using an exact algorithm 24 that locates the critical line $h_{c}^{r}(x)$ (i.e. the ultimate pinned configuration). Fig. 11 schematizes the model: we keep $h$ as a continuous variable and discretize the coordinate $x \rightarrow i$; periodic boundary conditions are used so that $h$ has period $M$ and $i$ has period $L$ [24, 25]. For a periodic disorder $M$ is kept constant as $L$ goes to infinity. In this case the interface winds around the system and feels the disorder periodicity. For a random manifold both $L$ and $M$ go to infinity. The most physical scaling corresponds to $M \sim L^{\zeta}$, where $\zeta$ is the roughness exponent at the depinning transition, and allows the transversal dimension to scale with the lateral extension of the interface 8, 11].

A general theorem for disorder-controlled transitions, shows that on a finite-volume $\left(L^{d+\zeta}\right)$, any divergent correlation length scales with an exponent [8, 11, 26]

$$
\nu_{\mathrm{FS}} \geq \frac{2}{d+\zeta} .
$$

The identification between $\nu_{\mathrm{FS}}$ and the critical correlation length exponent $\nu$ is a very delicate problem that has been discussed in the literature 27]. In any case, for elastic systems it was determined that $9,11,12,13]$,

$$
\sigma_{f_{c}}^{2} \equiv \overline{\left|f_{c}^{r}-\overline{f_{c}^{r}}\right|^{2}} \sim L^{-\frac{2}{\nu_{\mathrm{FS}}}},
$$

where the over-bar indicates disorder average.

Let us start discussing the periodic case, where by definition $\zeta=0$. We have performed multiple simulations
$M=$ const.

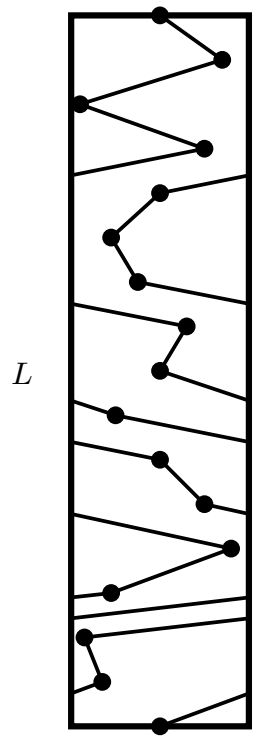

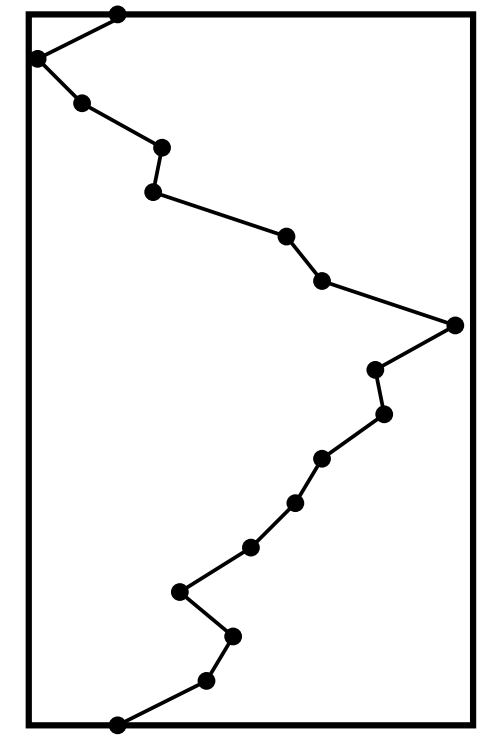

$M=k L^{\zeta}$
FIG. 1: Elastic lines in a disordered medium. The left schema illustrates a periodic system whereas the right one shows a random manifold.

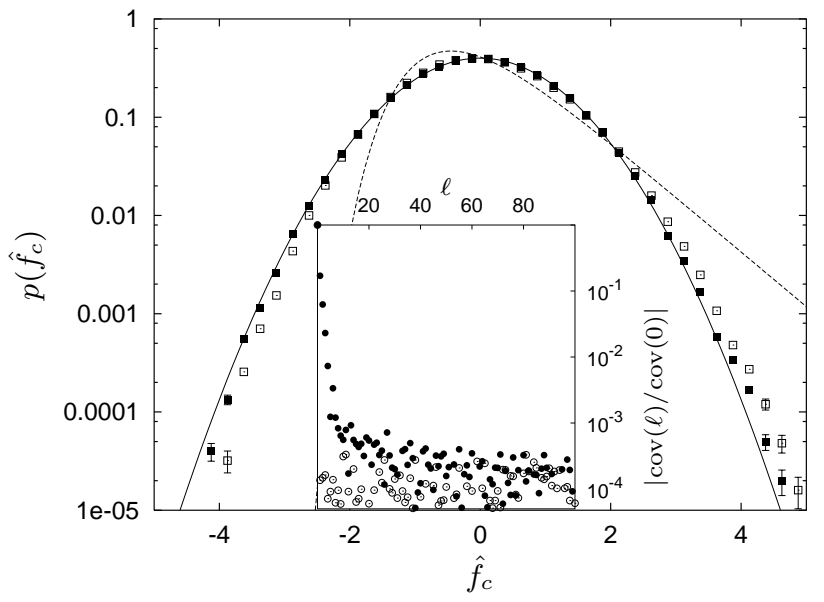

FIG. 2: Rescaled distributions for a periodic system. The squares correspond to the results for a periodic system $(M=$ 8) with $L=64$ (open) and $L=1024$ (filled); undisplayed error bars are smaller than the symbol size. The lines are the Normal (solid) and Gumbel (dashed) distributions. Convergence with $L$ towards the Gaussian limit is evident. The values for the skewness $\left(\gamma_{1}<0.03\right)$ and kurtosis $\left(\gamma_{2}<0.01\right)$ are consistent with this limit. Inset: covariance of the $\eta\left(i, h_{c}^{r}\right)$ 's (filled) compared with that of a set of random numbers (open).

for $d=1$, determining the critical force for different disorder realizations. We verify that the scaling relation given in Eq. (4) is obeyed with $\nu_{\mathrm{FS}} \simeq 2$ (in agreement with the results of previous simulations 9$]$ ). In order to highlight the universal behavior in the thermodynamic limit, it is convenient to rescale the forces and compare always zero-mean unit-variance distributions, $p\left(\hat{f}_{c}\right)$ with $\hat{f}_{c}=\left(f_{c}^{r}-\overline{f_{c}^{r}}\right) / \sigma_{f_{c}}$. In Fig. 2 we show that the limit distribution of $p\left(\hat{f}_{c}\right)$ is Gaussian. Finite size effects are very small and the limiting behavior is robust against changes in the nature of the disorder, the period $M$, the boundary conditions or any microscopic parameter like the elastic constant (in all figures, the number of samples ranges in $\left.10^{5}-10^{6}\right)$. Simulations done for $d=2$ also confirm this scenario. The generic nature of this result suggests that, in all periodic systems, the limiting distribution of thresholds (critical field, current, etc.) is Gaussian.

To understand this result, we study the correlation of the critical disorder-forces, $\eta\left(i, h_{c}^{r}\right)$, defined as the pinning forces on the different points of the critical line. Since the configuration is static, the critical force is obtained as minus the average pinning force. The degree of correlation among the $\eta\left(i, h_{c}^{r}\right)$ 's plays thus a defining role in determining the final thermodynamic distribution of critical forces. Evidently, if they were uncorrelated, the distribution would surely be Gaussian, on the general grounds of the central limit theorem. We studied therefore their covariance, defined as

$$
\operatorname{cov}(\ell)=\frac{1}{L} \sum_{i} \overline{\left(\eta\left(i+\ell, h_{c}^{r}\right)+\overline{f_{c}^{r}}\right)\left(\eta\left(i, h_{c}^{r}\right)+\overline{f_{c}^{r}}\right)} .
$$




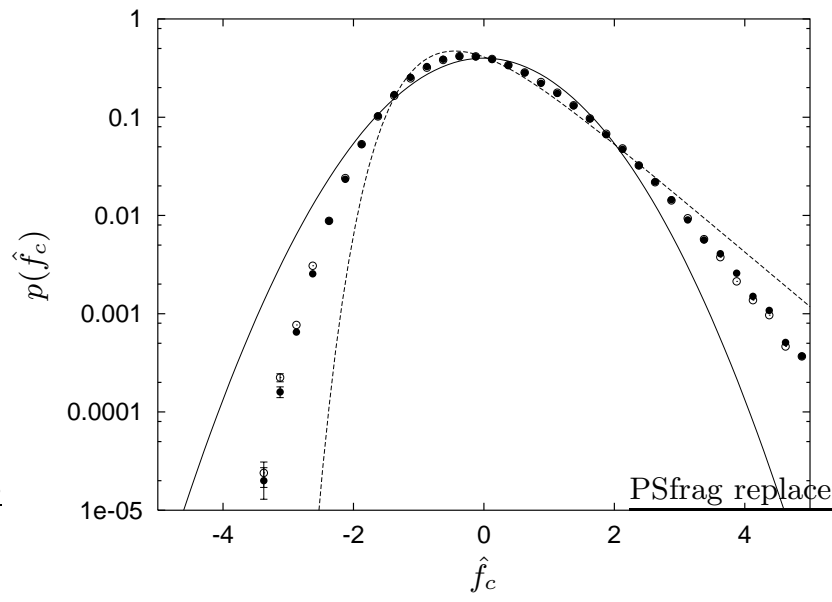

FIG. 3: Rescaled distributions for a random manifold. The circles correspond to the results for a scaling with $M=L^{\zeta}$ for $L=64$ (open) and $L=768$ (filled). The lines and error bars are as in Fig. 2 Convergence with $L$ towards a non-trivial limit is evident. The extrapolated values for the skewness and kurtosis are $\gamma_{1} \rightarrow 0.57 \pm 0.01$ and $\gamma_{2} \rightarrow 0.63 \pm 0.03$.

We found that, in the case of fixed finite $M$, the correlation decreases very fast (at least exponentially) and the variables quickly become uncorrelated (see the inset in Fig. 21), explaining the observed convergence of $p\left(\hat{f}_{c}\right)$ towards a Gaussian distribution. The $\eta\left(i, h_{c}^{r}\right)$ 's selfaverage in the thermodynamic limit, in turn implying that $\nu_{\mathrm{FS}}=2 / d$, saturating the bound of Eq. (3).

We direct now our attention to the case of random manifolds. We find $\nu_{\mathrm{FS}}=1.33 \pm 0.01$, verifying for $d=1$ the scaling relation $\nu_{\mathrm{FS}}=\nu=(2-\zeta)^{-1}$ [10, 11, 13], with $\zeta \sim 1.26 \pm 0.01[25,28]$. We stress that this relation is valid, as will be clearly shown later, only in correspondence to the physical scaling $M \sim L^{\zeta}$ (cf. Ref. [29]). In this case the distribution is non-trivial; our results for a $d=1$ random manifold are presented in Fig. 3 We find a distribution that converges as $L \rightarrow \infty$ to a universal curve that clearly deviates from the Gaussian in the direction of the Gumbel. Previous studies on cellular automaton models have found that distributions of nearthreshold depinning forces are also universal [30, 31].

The proper scaling for the random manifold case should be defined with care. We consider $M=k L^{\zeta}$ with $k$ a constant independent of $L$. For each fixed $k$, we find that the distribution of critical forces has a non-trivial thermodynamic limit. In particular, for $k \rightarrow 0$ this limit tends to a Gaussian, as seen in the periodic case, and in the opposite limit $(k \rightarrow \infty)$ the distribution approaches Gumbel's. We verified this behavior for $d=1$ and results for $d=2$ confirm the same scenario. The most natural definition of a random manifold system corresponds to considering values around certain, model dependent, $k_{w}=w / L^{\zeta}$ (where $w$ is the average width of the elastic line 32]). The ratio $k / k_{w}$ then parameterizes a universal

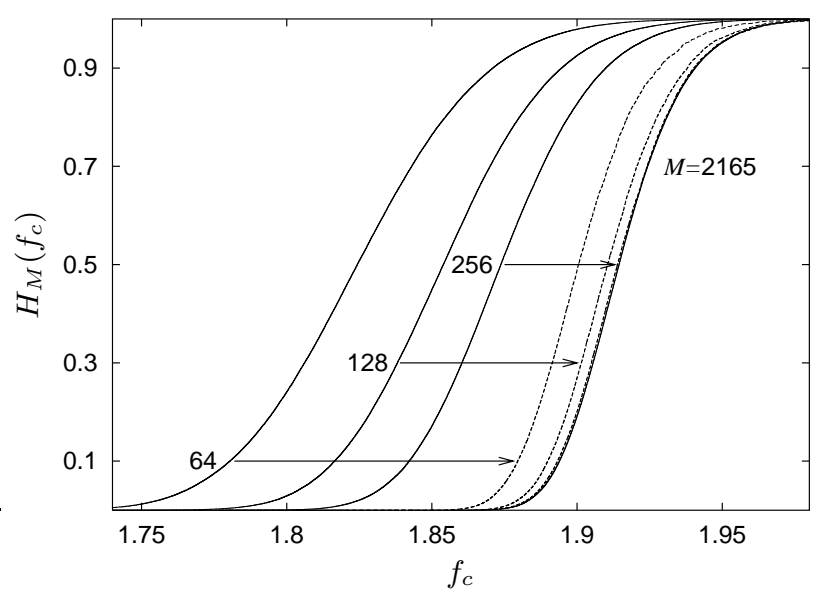

FIG. 4: Cumulative distributions of critical forces of lines with $L=256$. Different solid lines correspond to different $M$ 's as indicated. The average width is $w \sim 100\left(k_{w} \sim 0.1\right)$. The rescaling according to Eq. (6) of the curves for $M=$ $64,128,256$ to $M=2165(k=2)$, is shown with dashed lines.

family of functions that crosses over from the Gaussian to the Gumbel distribution. This set of extremal distributions is universal in the sense of being independent of all microscopic parameters of the model and fixed solely by $d$ and the ratio $k / k_{w}$.

To understand the Gumbel limit, let us start from the problem of iid variables. Given a set of $n$ variables with $Z_{n}$ its maximum, the associated cumulative probability distribution, $H_{n}(z)=P\left(z>Z_{n}\right)$, obeys:

$$
H_{n}(z)=\left[H_{m}(z)\right]^{\frac{n}{m}} .
$$

This is the crucial property that allows to prove the theorem on the statistics of extreme values mentioned at the beginning. We can now go back to the case of random manifolds. When the aspect ratio of the samples becomes very wide, $k \gg k_{w}$, the system can be thought to be split into $k / k_{w}$ approximately independent regions. This is because the configurations with non-zero depinning force proliferate and their correlation decreases. In this case Eq. (6) is asymptotically verified and the results of the theorem are still valid as shown by the statistics of extremes of stationary sequences [14, 15]. In order to illustrate this point, we study explicitly the cumulative probability of critical forces, $H_{M}\left(f_{c}\right)$. We present

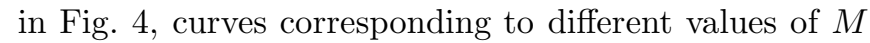
for $L$ large enough that the finite size effects are indistinguishable in the scale of the figure. We used Eq. (6) to scale the distributions towards one corresponding to a much larger $M$. Let us separate our discussion in three regimes: $k<k_{w}, k \gtrsim k_{w}$ and $k \gg k_{w}$. The figure shows that whereas in the first case the scaling of Eq. (6) is violated, in the other two it is obeyed with increasingly better accuracy as $k$ grows. This demonstrates that, in the third case, the critical force can be thought of as the 
maximum among forces sampled from a distribution like that in Fig. 3. Since the tail behavior of the latter is bounded between Gumbel and Gaussian (both belonging to the domain of attraction of the Gumbel distribution [15]), it follows that in the $k \rightarrow \infty$ limit the distribution should be Gumbel, as opposed to Fréchet or Weibull.

Let us discuss briefly the implications for other scalings of $M$. At this stage it should be clear that, for any other scaling, the distribution converges to a nonparametric thermodynamic limit. Consider, for the sake of concreteness, $M \sim L^{\zeta^{\prime}}$ : on the one hand, for $\zeta^{\prime}>\zeta$, the convergence is towards the Gumbel distribution; on the other hand, for $\zeta^{\prime}<\zeta$, the limit is the Gaussian.

As we have seen, the study of the distributions of maximum thresholds unravels a very rich and complex scenario. For all periodic systems the distribution is a Gaussian, - a non-trivial result from the viewpoint of the theory of extreme value statistics. This result rests on the asymptotic validity of the central limit for the critical depinning forces and implies $\nu_{\mathrm{FS}}=2 / d$. For random manifolds the situation is more intricate and there exists a universal family that interpolates continuously from the Gaussian to the Gumbel distribution. To our knowledge, this is the first time a set of distributions of extremes with these characteristics was identified, (there is another recent example of a family that interpolates between these two limits, but with no interpretation in terms of extremes 33]). The criticality of the system is crucial to obtain such varied behaviors. Indeed, it is the infinite range of the correlations that build up along the critical manifold that is responsible for the strong deviations from the standard theory for iid variables.

It would be extremely interesting to verify these scenarios experimentally. We believe, good candidate measurements in periodic systems would be those of superconducting critical currents, or charge density waves. For propagating interfaces the usual set-ups involve $M \ggg w$. To build a distribution in this case one should be able to measure the critical thresholds for a given tiling of the sample, then compare distributions among different tiling widths. On a different line, we already started to look into the analytical characterization of the family of distributions that we found for the random manifold case. This might provide deep insights into the theory of extremes of correlated variables that is still in its infancy.

We acknowledge the illuminating discussions with M. Droz, P. Le Doussal, W. Krauth, M. Mézard, Z. Ràcz and K. J. Wiese. We thank T. Giamarchi and W. Krauth for their comments on the manuscript.

[1] S. Lemerle et al., Phys. Rev. Lett. 80, 849 (1998).

[2] T. Tybell, P. Paruch, T. Giamarchi, and J. M. Triscone, Phys. Rev. Lett. 89, 097601 (2002).
[3] S. Moulinet, A. Rosso, W. Krauth, and E. Rolley, Phys. Rev. E 69, 035103(R) (2004).

[4] E. Bouchaud, J. P. Bouchaud, D. S. Fisher, S. Ramanathan, and J. R. Rice, J. Mech. Phys. Solids 50, 1703 (2002).

[5] G. Grüner, Rev. Mod. Phys. 60, 1129 (1988).

[6] G. Blatter et al., Rev. Mod. Phys. 66, 1125 (1994).

[7] R. Chitra, T. Giamarchi, and P. Le Doussal, Phys. Rev. B 65, 035312 (2001).

[8] P. Chauve, P. Le Doussal, and K. J. Wiese, Phys. Rev. Lett. 86, 1785 (2001).

[9] A. A. Middleton and D. S. Fisher, Phys. Rev. B 47, 3530 (1993).

[10] T. Nattermann, S. Stepanow, L. H. Tang, and H. Leschhorn, J. Phys. (Paris) 2, 1483 (1992).

[11] O. Narayan and D. S. Fisher, Phys. Rev. B 48, 7030 (1993).

[12] O. Narayan and D. S. Fisher, Phys. Rev. B 46, 11520 (1992).

[13] P. Le Doussal, K. J. Wiese, and P. Chauve, Phys. Rev. B 66, 174201 (2002).

[14] S. Coles, An Introduction to the Statistical Modeling of Extreme Values (Springer, London, 2001).

[15] J. Galambos, The Asymptotic Theory of Extreme Order Statistics (Krieger Publishing, Malabar, Florida, 1987).

[16] J.-P. Bouchaud and M. Mézard, J. Phys. A 30, 7997 (1997).

[17] D. Carpentier and P. Le Doussal, Phys. Rev. E 63, 026110 (2001).

[18] P. Le Doussal and C. Monthus, Physica A 317, 140 (2003).

[19] G. Györgyi, P. C. W. Holdsworth, B. Portelli, and Z. Rácz, Phys. Rev. E 68, 056116 (2003).

[20] D. S. Dean and S. N. Majumdar, Phys. Rev. E 64, 046121 (2001).

[21] A. A. Moreira, J. S. Andrade, and L. A. N. Amaral, Phys. Rev. Lett. 89, 268703 (2002).

[22] A. A. Middleton, Phys. Rev. Lett. 68, 670 (1992).

[23] A. Rosso and W. Krauth, Phys. Rev. B 65, 012202 (2002).

[24] A. Rosso and W. Krauth, Phys. Rev. E 65, R025101 (2002).

[25] A. Rosso, A. K. Hartmann, and W. Krauth, Phys. Rev. E 67, 021602 (2003).

[26] J. T. Chayes, L. Chayes, D. S. Fisher, and T. Spencer, Phys. Rev. Lett. 57, 2999 (1986).

[27] F. Pázmándi, R. T. Scalettar, and G. T. Zimányi, Phys. Rev. Lett. 79, 5130 (1997).

[28] H. Leschhorn and L. Tang, Phys. Rev. E 70, 2973 (1993).

[29] S. Ramanathan and D. S. Fisher, Phys. Rev. B 58, 6026 (1998).

[30] R. Skoe, D. Vandembroucq, and S. Roux, Int. J. Mod. Phys. C 13, 751 (2002), see also arXiv:cond-mat/0311485

[31] J.-C. Baret, D. Vandembroucq, and S. Roux, Phys. Rev. Lett. 89, 195506 (2002).

[32] A. Rosso, W. Krauth, P. Le Doussal, J. Vannimenus, and K. J. Wiese, Phys. Rev. E 68, 036128 (2003).

[33] T. Antal, M. Droz, G. Györgyi, and Z. Rácz, Phys. Rev. E 65, 046140 (2001).

[34] We recall that the Gumbel distribution is given by $(\pi / \sqrt{6}) g(x) \exp (-g(x))$, where $g(x)=\exp (-\pi x / \sqrt{6}-\gamma)$ and $\gamma$ is Euler's constant. 